\title{
Oxigenoterapia hiperbárica em lesões actínicas de colo de ratos. Aspectos morfológicos e morfométricos ${ }^{1}$
}

\author{
Hiperbaric oxygen in actinic lesions of rat colon. Morphological and morphometric as- \\ pects
}

\author{
Maria Ligia Lyra Pereira², Tânia Cilja Scheidt ${ }^{3}$, Manuel de Jesus Simões ${ }^{4}$, Rejane Mosquette ${ }^{5}$, Paulo de Oliveira Gomes ${ }^{6}$ \\ 1. Trabalho realizado na Faculdade de Ciências Médicas de Santos, Universidade Lusíadas (FCMS-UNILUS). Laureado com o Prêmio "Pitanga \\ Santos" no $53^{\circ}$. Congresso de Coloproctologia, Campos do Jordão, set. 2004. \\ 2. Professora Auxiliar de Ensino da Disciplina de Cirurgia Geral do Departamento de Cirurgia da UNILUS \\ 3. Pós-Graduanda do Programa de Cirurgia e Experimentação da UNIFESP-EPM. \\ 4. Professor Livre Docente da Disciplina de Histologia e Biologia Estrutural do Departamento de Morfologia da UNIFESP-EPM. \\ 5. Pós-Graduanda do Programa de Morfologia da UNIFESP-EPM. \\ 6. Professor Adjunto da Disciplina de Técnica Operatória e Cirurgia Experimental da UNIFESP-EPM.
}

\section{RESUMO}

Objetivo: Avaliar a ação da oxigenação hiperbárica em lesões actínicas provocadas por radiação ionizante no colo distal de ratas. Métodos: Foram utilizadas 32 ratas adultas distribuídas em quatro grupos. I - sham; II - oxigenoterapia hiperbárica (OHT); III radioterapia (RT), e IV - radioterapia e oxigenoterapia hiperbárica (da mesma maneira que nos grupos II e III). Segmentos de colo distal foram fixados em formol a 10\% e processados para avaliação histológica e morfométrica. Resultados: Os resultados do experimento mostraram lesão das glândulas intestinais, vasodilatação e infiltrado leucocitário na lâmina própria e submucosa no colo dos animais pertencentes ao grupo RT que diminuiu acentudadamente após a oxigenioterapia hiperbárica. Conclusão: A oxigenitoterapia hiperbárica atenua as alterações morfológicas da mucosa e submucosa do cólon distal de ratas com enterite induzida por radiação ionizante.

Descritores: Oxigenação hiperbárica. Radioterapia. Cólon. Ratos.

\section{ABSTRACT}

Purpose: To evaluate the effects of hiperbaric oxygen on the radiation-induced actinic lesions in the rat distal colon. Methods: Thirty-two Wistar female rats were divided into four groups: I - sham; II - hiperbaric oxygen therapy (HOT); III - radiotherapy (RT), e IV - radiotherapy and hiperbaric oxygen (the same manner of II and III group). Fragments of distal colon were fixed into $10 \%$ formadehyde and proceeded for the histological routine and morphology. Results: The experimental results showed the intestinal gland lesions, vasodilation and infiltrated leucocytes in propria laminae and submucosae in rat colon of GIII that accentuated colon after hiperbaric oxygen Conclusion: Hiperbaric oxygen attenuated the morphological changes in the mucosae and submucosae of the rat distal colon with radiation-induced inflammatory process.

Key words: Hyperbaric Oxygenation. Radiotherapy. Colon. Rats.

\section{Introdução}

A radioterapia tem importância no tratamento dos tumores pélvicos como fator curativo, adjuvante ou paliativo. A adequação do tratamento é traduzida pelo prolongamento do tempo de vida com concomitante diminuição no índice de recidiva local da doença ${ }^{1}$.

No entanto, os efeitos colaterais precoces e tardios da radiação sobre os órgãos pélvicos próximos ao órgão alvo são fatores que comprometem a qualidade de vida do paciente. Portanto, os cuidados para diminuir estes efeitos são tão relevantes quanto sua indicação e utilização precisas ${ }^{2,3}$.
Os sintomas decorrentes das lesões actínicas colorretais podem ser classificados em agudos (primeiras manifestações entre dois e seis meses) e crônicos (manifestações entre seis meses e dois anos). No primeiro grupo, a ocorrência mais freqüente consiste em um aumento no número de evacuações, estando a intensidade diretamente relacionada à dose, ao tempo (freqüência e duração) e ao volume de massa corpórea irradiada. No segundo grupo, as estenoses, hemorragias, perfurações agudas e fístulas sempre foram a grande preocupação e dependem da dose total recebida, do volume total de tecido 
irradiado e da radiossensibilidade do paciente ${ }^{4,5}$.

Os pacientes portadores de lesão retal induzida por radioterapia podem ser submetidos a tratamento medicamentoso sintomático com o emprego de anticolinérgicos, antiespamódicos e analgésicos. Na dependência da intensidade e duração dos sintomas, medicamentos como antibióticos, prostaglandinas, repositores da flora intestinal e dietas à base de glutamina, podem auxiliar no controle e remissão da doença. O uso de corticóides via parenteral ou por enema tem indicação nos casos de sintomas mais intensos. Recentemente, tratamentos invasivos como a fotocoagulação a laser são também empregados ${ }^{6,7}$.

As intervenções operatórias para desvio de trânsito como a colostomia proximal à lesão, a mucossectomia, a ressecção retal seguida de anastomose coloanal, ou amputação abdominoperineal do reto, são outras opções para os casos refratários ao tratamento medicamentoso ${ }^{8}$.

Estudos clínicos demonstraram que pacientes portadores de cistite hemorrágica e enterorragia grave de origem actínica submetidos a oxigenação hiperbárica (OHB), associados ou não ao tratamento tradicional, apresentaram boa evolução com diminuição ou desaparecimento do sangramento $9,10,11$.

A oxigenoterapia hiperbárica seria uma das opções de diminuir os efeitos adversos da radioterapia, sem os efeitos colaterais de alguns medicamentos utilizados no tratamento clínico tradicional, os riscos das terapêuticas invasivas e as alterações de função decorrentes das intervenções operatórias ${ }^{9}$.

Trabalho experimental, onde foi analisado o íleo terminal de ratos submetidos à radiação, demonstrou o efeito protetor da OHB. Os animais foram expostos a oxigenoterapia depois de completada a dose total de radiação. Ocorreu diminuição significante das lesões recentes e tardias, quando se comparou o grupo com ou sem exposição a $\mathrm{OHB}{ }^{12}$.

Deve ser mencionado que a ação da $\mathrm{OHB}$ potencializa o efeito radiobiológico, quando realizada concomitante às sessões de radioterapia 13 . O aumento na oferta de oxigênio amplia a produção de radicais livres, com exacerbação da lesão oxidativa do ácido desoxirribonucléico (DNA) e agravamento no índice de morte celular ${ }^{14}$.

Por outro lado, a OHB promove também aumento da produção de óxido nítrico, com conseqüente vasodilatação local, o que diminuiria a isquemia na fase aguda da aplicação ionizante e seria um dos mecanismos de diminuição de fenômenos apoptóticos, sendo, deste modo, um fator de proteção às lesões radioinduzidas ${ }^{15}$.

A revisão de literatura é escassa na relação entre o tratamento de lesões intestinais actínicas com OHB. Apesar de existir um grande número de artigos publicados sobre o assunto, a maioria dos trabalhos realizados resume-se a descrições de casos e ensaios clínicos não controlados ou com controles inadequados, o que fornece pouca evidência para sua indicação em diferentes situações clínicas ${ }^{9,11}$.

Portanto, o objetivo é avaliar as alterações morfológicas e morfométricas do cólon de ratos submetidos à oxigenação hiperbárica e à radiação ionizante.

\section{Métodos}

Foram utilizados ratos fêmeas Wistar, adultas (Rattus norvegicus albinus), com peso médio de 260 gramas, provenientes do Biotério da Faculdade de Ciências Médicas de Santos, Universidade Lusíadas (FCMS-UNILUS). Os animais foram alojados no referido Biotério, com água e ração ad libitum, iluminação controlada por ciclos com 12 horas claro e 12 horas escuro (período de luz das 7:00 às 19:00 horas), com temperatura e umidade controladas. Após um período de adaptação de 7 dias, os animais foram distribuídos aleatoriamente em quatro grupos, a saber: Grupo Sham - três animais anestesiados e submetidos à ressecção de um segmento de $2 \mathrm{~cm}$ de colo distal; Grupo Radioterapia (RT) - dez animais submetidos a oito sessões de radioterapia (60 Gy), os quais, após sete dias, foram anestesiados e submetidos à ressecção de um segmento de $2 \mathrm{~cm}$ de colo distal; Grupo Oxigenoterapia Hiperbárica (OHB) - nove animais submetidos a sessões diárias de oxigenação hiperbárica por cinco dias, os quais, após três dias, foram anestesiados e submetidos à ressecção de um segmento de $2 \mathrm{~cm}$ de colo distal; Grupo Radioterapia e Oxigenoterapia Hiperbárica (RT + OHB) - dez animais submetidos a oito sessões de radioterapia (60 Gy), os quais, após sete dias da última aplicação, foram expostos a sessões diárias de OHB por cinco dias; após três dias, foram anestesiados e submetidos à ressecção de um segmento de $2 \mathrm{~cm}$ de colo distal.

Todos os procedimentos cirúrgicos foram realizados no Laboratório de Técnica Cirúrgica da FCMS-UNILUS, após aprovação prévia do Projeto de Pesquisa pelo Comitê de Ética em Pesquisa (CEP) do Hospital São Paulo - UNIFESP-EPM e protocolado sob o número 1022/03 e ratificado pela FCMSUNILUS.

A oxigenação hiperbárica foi realizada em câmara hiperbárica de acrílico para animais de pequeno porte, a três atmosferas absolutas (ATA) por 90min por dia, durante cinco dias. Após três dias de observação, foram submetidos ao mesmo procedimento descrito para o Grupo Sham.

A radioterapia foi realizada na Sociedade Portuguesa de Beneficência de Santos, delimitando a exposição superior à cicatriz umbilical e inferior às espinhas isquiáticas. Para tanto, os animais foram submetidos a oito sessões de 5Gy, obtidos por exposição a aparelho de ortovoltagem*. Deste modo, foi atingida dose total de 60Gy na superfície do animal e de 54Gy no fragmento de colo a ser estudado.

Ao final do experimento todos os animais foram anestesiados com zolazepam em combinação com tiletamina na dose de $50 \mathrm{mg} \cdot \mathrm{kg}^{-1}$, associando-se analgesia com cloreto de petidina a 0,2mg.kg-1 em uma única aplicação por via intramuscular. Em seguida, os animais foram submetidos a laparotomia, identificado o cólon distal com limite cranial correspondente à projeção da primeira vértebra sacral e caudal à junção vesicouretral. Este segmento, com aproximadamente $2 \mathrm{~cm}$, foi retirado e imediatamente mergulhado em solução contendo formol a $10 \%$.

Os animais do Grupo RT+OHB foram submetidos ao mesmo 
processo de exposição à radioterapia descrito para o grupo RT e, em seguida, observados por sete dias. No oitavo dia foram expostos a oxigenação hiperbárica por cinco dias consecutivos e, após três dias, submetidos ao mesmo procedimento operatório já descrito para os animais do grupo OHB.

Os fragmentos do cólon foram fixados em formol a $10 \%$ e processados segundo as técnicas histológicas para inclusão em parafina. Cortes com $3 \mu \mathrm{m}$ de espessura foram corados pela hematoxilina e eosina (H.E).

A análise morfométrica foi realizada avaliando-se a proporção de vasos sanguineos por 40 campos, com aumento final de 360 vezes. Para tanto, foi utilizada uma ocular integradora Kpl 8x da Carl Zeiss contendo retículo de Weibel de 25 pontos. Foram contados apenas os pontos que incidiram sobre os vasos sanguíneos. Em seguida, foi aplicado o teste da análise de variância de Kruskal-Wallis $(\alpha \leq 0,05)$.

\section{Resultados}

\section{Morfológicos}

No momento da retida dos segmentos de cólon nos animais irradiados (RT) observou-se presença de coloração intensamente avermelhada com hiperemia. Nos animais do grupo $\mathrm{RT}+\mathrm{OHB}$, os segmentos apresentaram as mesmas alterações, porém em menor intensidade.

Grupo Sham: A mucosa apresentou-se revestida por um epitélio cilíndrico simples, sendo rica em células caliciformes e em células absortivas. A lâmina própria estava constituída por tecido conjuntivo frouxo onde havia inúmeras glândulas tubulares retas ricas em células caliciformes. Abaixo do fundo das glândulas percebia-se nitidamente a presença da muscular da mucosa, a qual delimitava a mucosa da submucosa. A submucosa estava constituída por tecido conjuntivo denso, vasos sanguíneos, linfáticos e nervos. A muscular mostrou-se formada basicamente por fibras musculares lisas dispostas em duas camadas, uma circular interna e uma longitudinal externa. Por fora do tubo digestório, notou-se a presença de uma camada de células pavimentosas apoiadas sobre uma lâmina de tecido conjuntivo fazendo parte da serosa (Figura 1).

Grupo RT: neste grupo o intestino grosso apresentou constituição morfológica semelhante à do grupo Sham, no entanto, notou-se no local submetido à irradiação áreas congestas e hemorrágicas. Em alguns animais observou-se desintegração da mucosa com descamação do epitélio superficial (Figuras 1 e 2). Observaramse ainda no epitélio superficial e de revestimento inúmeras células com núcleos heterocromáticos, com sinais de apoptose. Na lâmina própria e na submucosa apresentaram-se também inúmeros vasos sanguíneos dilatados.

Grupo OHB: o intestino grosso apresentou os mesmos aspectos observados no grupo Sham (Figura 1).

Grupo RT+OHB: notaram-se em alguns animais, na área submetida á irradiação, algumas células epiteliais em descamação. A lâmina própria e a submucosa apresentaram também inúmeros vasos sanguíneos dilatados (Figura 1).
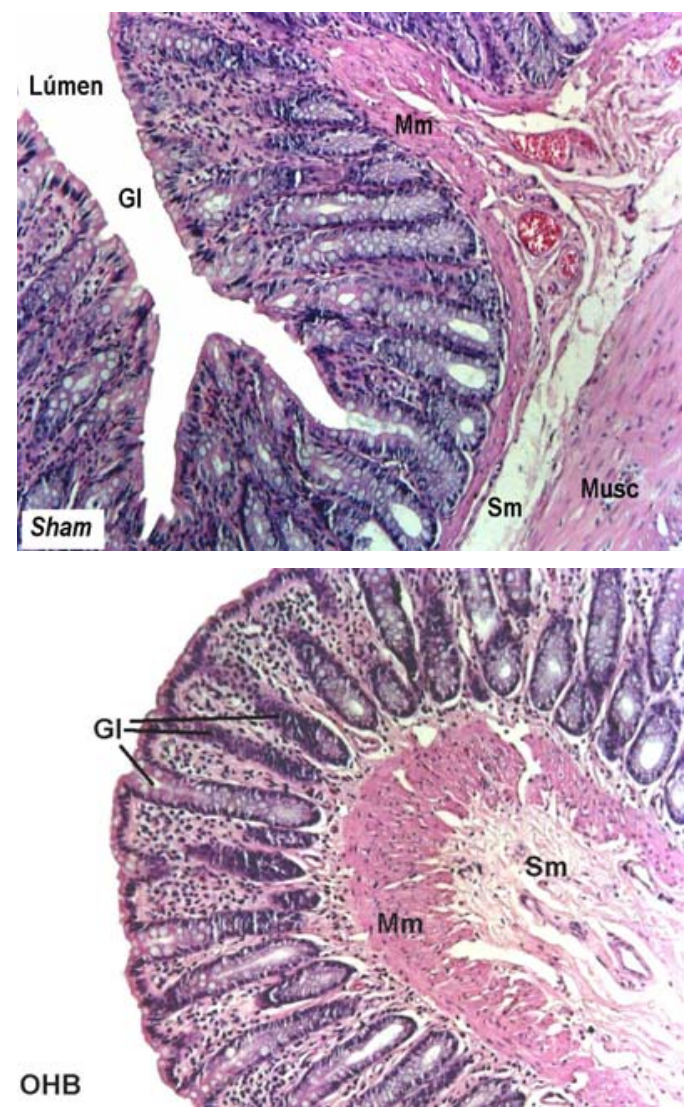
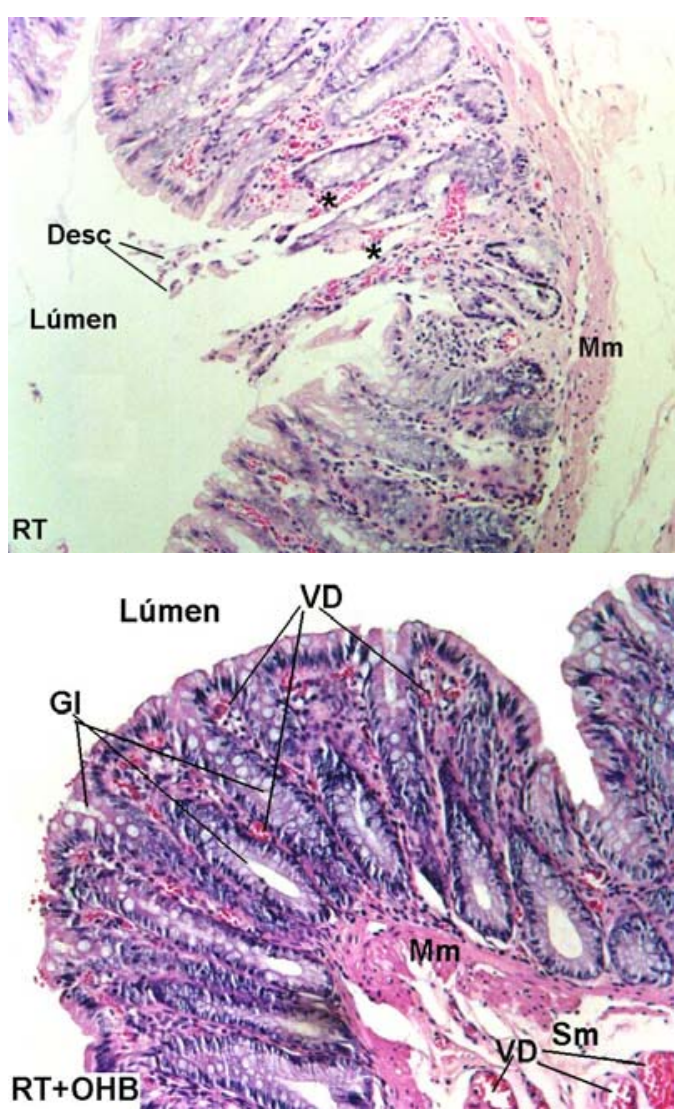

FIGURA 1 - Fotomicrografia que mostra porções do intestino grosso de ratas pertencentes aos vários grupos de estudo: Sham, $\mathrm{RT}$ - radioterapia, OHB oxigenação hiperbárica e, RT + OHB - radioterapia associada a oxigenação hiperbárica. Notar em RT células descamadas (Desc) para o interior do lúmen e áreas de hemorragia $(*)$. Em RT + OHB observar preservação das glândulas (Gl) e vasodilatação (VD). Mm - muscular da mucosa e Sm - submucosa. H.E. $230 \mathrm{X}$ 


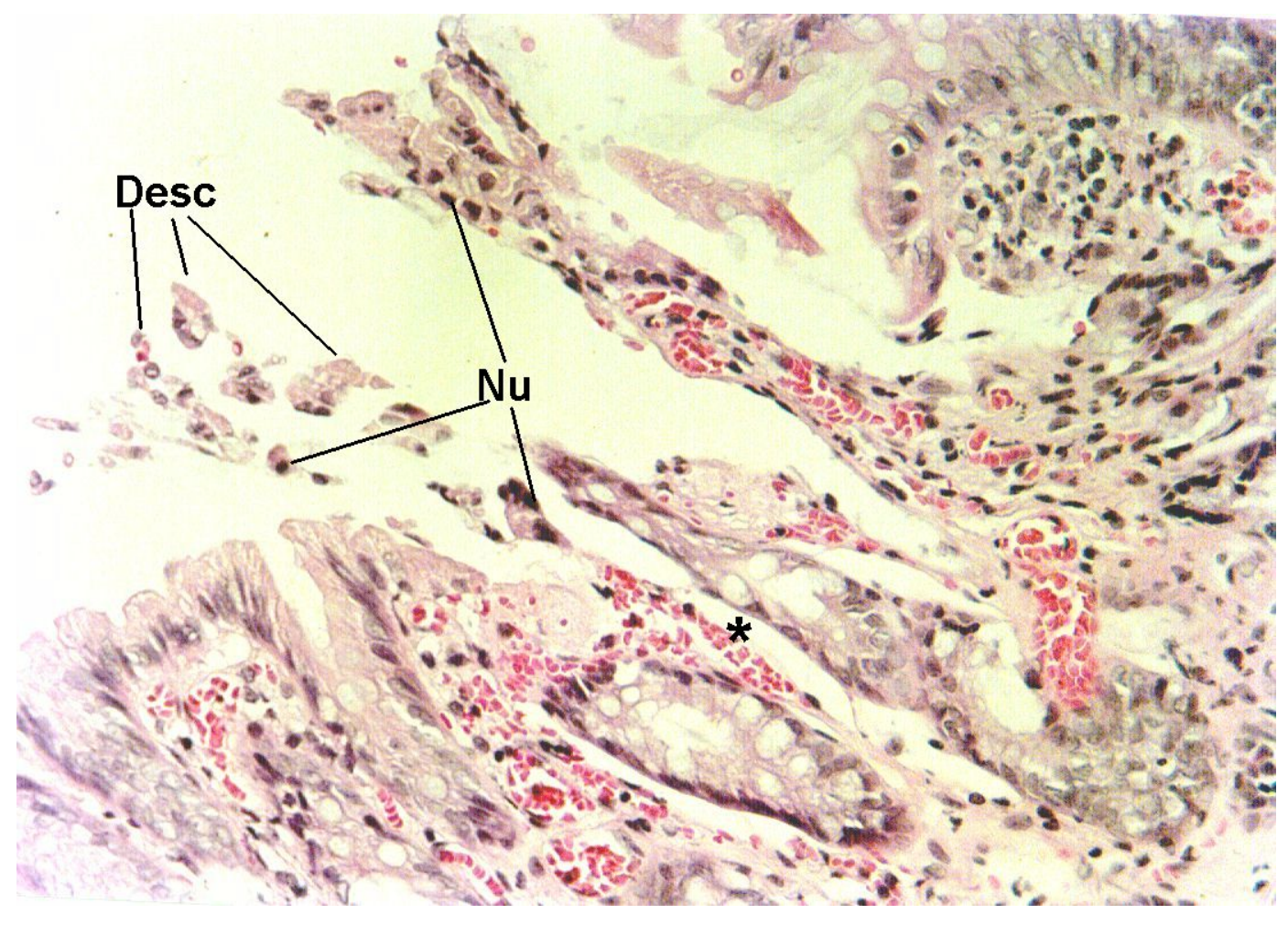

FIGURA 2 - Fotomicrografia que mostra detalhe do intestino grosso de rata pertencente ao grupo submetido à radioterapia. Notar células descamadas (Desc), áreas hemorrágicas (*) e núcleos heterocromáticos $(\mathrm{Nu})$. H.E. $420 \mathrm{X}$

TABELA 1 - Avaliação morfológica do grau de lesão de criptas, dilatação vascular e infiltrado leucocitário na lâmina própria e submucosa nos vários grupos estudados

\begin{tabular}{lcccc}
\hline \multirow{2}{*}{ Parâmetros } & \multicolumn{4}{c}{ Grupos } \\
\cline { 2 - 5 } Dilatação vascular & Sham & OHB & RT & RT+OHB \\
Lâmina própria & - & - & +++ & + \\
Submucosa & - & - & +++ & + \\
\hline Infiltrado leucocitário & & & & \\
Lâmina própria & $+/-$ & $+/-$ & +++ & + \\
Submucosa & - & - & +++ & + \\
\hline Lesão de criptas & - & - & +++ & + \\
\hline
\end{tabular}

Legenda: $\mathrm{OHB}=$ oxigenação hiperbárica, $\mathrm{RT}=$ radioteratipa.

Graus de lesão: ausente(-), leve(+), moderada(++) e intensa(+++). 


\section{Morfométricos}

Os valores obtidos pela análise morfométrica estão expressos na tabela 2.

TABELA 2 - Valores médios da proporção de vasos sanguíneos observados no cólon distal de ratas nos vários grupos de estudo

\begin{tabular}{ccccc}
\hline & \multicolumn{5}{c}{ Grupos } \\
\cline { 2 - 5 } & Sham & OHB & RT & RT+OHB \\
\hline Média & 3,15 & 4,42 & 18,52 & 7,52 \\
\cline { 2 - 5 } EPM & 0,24 & 0,36 & 0,66 & 0,70 \\
\hline
\end{tabular}

Legenda: $\mathrm{OHB}=$ oxigenação hiperbárica, $\mathrm{RT}=$ radioteratipa $\mathrm{EPM}=$ Erro Padrão da Média

Análise de Kruskal-Wallis

$\mathrm{H}$ calculado $=101,63 *$ H crítico $=7,82$

$(\mathrm{P}=0,001)$

$\mathrm{RT}>$ Simulado e $\mathrm{OHB}$ e $\mathrm{RT}+\mathrm{OHB}$

\section{Discussão}

Este trabalho apresenta um modelo de lesão actínica de intestino grosso de ratas e associa uma opção terapêutica, a oxigenação hiperbárica (OHB), que pelas suas características teóricas de modo de ação, poderia proteger as células intestinais dos efeitos deletérios da radiação.

A OHB provoca aumento das taxas de oxigenação tecidual e já foi utilizada concomitante às aplicações de radioterapia, pois amplifica em duas ou três vezes seu efeito nos tecidos tumorais, induzindo a um aumento da lesão celular produzida pelos radicais livres e maior efetividade do tratamento por radiação ionizante ${ }^{13}$.

Em contraposição, trabalhos clínicos demonstram o efeito benéfico da OHB em lesões actínicas de reto ${ }^{10,11,16,17}$. Estudo experimental em ratos avaliou alterações morfológicas e morfométricas de lesões actínicas tardias em íleo terminal submetidas precocemente à $\mathrm{OHB}$, confirmando seu efeito protetor $^{12}$.

Para a OHB, foi utilizada câmara hiperbárica de acrílico para animais de pequeno porte ${ }^{18}$, que os mantinha sem necessidade de anestesia ou analgesia, em condições adequadas para receber oxigênio a $100 \%$ em 3 ATA, sem intercorrências na fase de compressão ou descompressão ${ }^{19}$. Sua utilização foi observada em ratos submetidos à lesão isquemia-reperfusão, demonstrando a prevenção da ocorrência de lesão de mucosa quando aplicada durante a isquemia ${ }^{20}$.

O modelo de produção de enterite utilizado procurou simular uma situação que ocorre em seres humanos que necessitam submeter-se à radioterapia para tratamento de tumores pélvicos. Procurou-se estabelecer um paralelo entre a dose empregada de radiação e a distância do órgão-alvo. O modelo foi reforçado por relatos que estimam em $5 \%$ o risco de desenvolvimento de complicações em cinco anos com dose total entre 60 a $65 \mathrm{~Gy}^{14}$, embora outros já refiram aparecimento de lesão com o uso de 10 Gy. A escolha da dose usada foi semelhante a trabalho em ratos, que utilizou a OHB para tratamento de lesão actínica de íleo terminal ${ }^{12}$.

As manifestações da enterite começaram após a terceira ou quarta aplicação da radioterapia e cessaram cerca de quatro dias após a última aplicação. Diante destes achados, considerou-se que o modelo utilizado produziu um quadro agudo de enterite de intensidade leve a moderada.

Os animais do grupo de oxigenação hiperbárica tiveram descrição morfológica semelhante ao grupo de animais normais. A OHB não provocou nenhuma alteração estrutural apreciável, na avaliação por microscopia de luz, sobre as estruturas anatômicas do cólon de ratas.

As alterações notadas foram avaliadas do ponto de vista qualitativo em leves, moderadas e intensas, e, a tabela 3 expressa esses achados, mostrando que a radiação ionizante produziu alterações morfológicas compatíveis com um quadro de enterite actínica.

A contagem de vasos presentes nas camadas mucosa e submucosa mostra que a presença de vasos está significantemente aumentada nos animais irradiados, sendo mais uma tradução da resposta inflamatória à agressão da radiação e caracterizando o quadro de enterite actínica.

A avaliação dos animais submetidos à irradiação e oxigenoterapia hiperbárica no tocante aos aspectos histológicos mostrou que o quadro geral é semelhante aos dos animais submetidos á irradiação exclusiva. No entanto, o grau das alterações - hemorragia, descamação e vasodilatação é, qualitativamente, em menor intensidade. A quantidade de vasos 
nas camadas mucosa e submucosa é significantemente menor que no grupo irradiado exclusivamente, e, próxima aos dos grupos normal ou de oxigenoterapia hiperbárica.

A interpretação cabível é que a OHB promoveu, de algum modo, uma melhora na evolução das lesões induzidas pela radiação ionizante. O mecanismo de ação pelo maior aporte de oxigênio às áreas atingidas pela radiação pode ter melhorado, num primeiro instante, a isquemia a que normalmente está sujeita a área que recebe a radiação. A presença de vasodilatação presente nos animais irradiados pode ser a expressão compensatória da necessidade de aporte de substratos à área lesada na tentativa de remover o tecido necrótico e reparar a lesão. A ação do oxigênio favoreceria esta ação levando a uma recuperação mais rápida dos estágios inflamatórios, o que se traduziu por alterações menos intensas na avaliação morfológica.

Considerando a análise dos resultados obtidos, percebe-se que o modelo de enterite actínica proposto foi adequado para o estudo e pode ser utilizado em outros protocolos de pesquisa. A oxigenação hiperbárica mostrou uma participação benéfica discreta, a ser mais bem explorada em trabalhos futuros, sobre a evolução da enterite induzida por radiação.

\section{Conclusão}

A oxigenoterapia hiperbárica diminui as alterações morfológicas da mucosa e submucosa de colo distal de ratos com enterite induzida por radiação ionizante.

\section{Referências}

1. Goligher, JC. Cirurgia del ano, recto y colon. 3ed. Barcelona: Salvat; 1988.

2. Johnson RJ, Carrington BM. Pelvic radiation disease. Clin Radiol. 1992; 45:4-12.

3. Lichter AS, Lawrence TS. Recents advances in radiation oncology. N Eng J Med. 1995; 332:371-9.

4. Taylor PM, Johnson RJ, Eddleston B, Hunter RD. Radiological changes in the gastrintestinal and genitourinary tract following radiotherapy for carcinoma of the cervix. Clin Radiol. 1990; 41:1659.

5. Nussbaum ML, Campana TJ, Weese JL. Radiation-induced intestinal injury. Clin Plast Surg. 1993; 20:573-80.

6. Campos FG, Mucerino DR, Waitzberg DL, Logulo AF, El Ibrahim
R, Nadalin W, et al. Efeitos protetores da glutamina e dieta elementar na enterocolite actínica aguda: avaliação histológica. Rev Assoc Med Bras. 1994; 40:143-9.

7. Lee TK, Stupans I. Radioprotection: the non-steroidal antiinflamatory drugs (NSAIDs) and prostaglandins. J Pharm Pharmacol. 2002; 54:1435-45.

8. Allen-Mersh TG, Wilson MRCP, Hope-Stone HF, Mann CV. The management of late radiation-induced rectal injury after treatment of carcinoma of the uterus. Surg Gynecol Obstet. 1987; 164:521-4.

9. Nakada T, Kubota Y, Sasagawa I, Suzuki H, Yamaguchi T, Ishigooka $\mathrm{M}$; et al. Therapeutic experience of hyperbaric oxygenation in radiation colitis. Dis Colon Rectum. 1993; 36:962-5.

10. Kindwall EP. Hyperbaric oxygen treatment of radiation cystitis. Clin Plast Surg. 1993; 20:589-92.

11. Moulin C, Li V, Loizzo F, Faudemay C, Hostein J. Intérêt de l'oxygène hyperbare dans le traitement à visée hémostatique des rectosigmoïdites radiques chroniques. Gastroenterol Clin Biol. 1993; 17:520-1.

12. Feldmeier JJ, Da Volt DA, Court WS, Onoda JM, Alecu R. Histologic morphometry confirms a prophylatic effect for hyperbaric oxygen in the prevention of delayed radiation enteropathy. Undersea Hyper Med. 1998; 25:93-7.

13. Segreto HRC, Segreto RA. Revisão e atualização em radiobiologia. Aspectos celulares, moleculares e clínicos. Folha Med. 2000; 119:927.

14. Salvajoli JV, Souhami L, Faria SL. Radioterapia em oncologia. 1ed. São Paulo: Editora Médica e Científica Ltda.; 1999.

15. Bersimbaev RI, Yugai YE, Hanson PJ, Tzoy IG. Effect of nitric oxide on apoptotic activity in the rat gastrointestinal tract. Eur J Pharmacol. 2001; 423:9-16.

16. Miura M, Sasagawa I, Kubota Y, Ijima Y, Sawamura T, Nakada T. Effective hyperbaric oxygenation with prostaglandin E1 for radiation cystitis and colitis after pelvic radiotherapy. Intern Urol Nephrol. 1996; 28:643-7.

17. Dias MD. Aplicações clínicas do oxigênio hiperbárico. Diag Trat. 2001; 6:1-7.

18. Pereira MLL, Teles AP, Pereira Neto J. Câmara hiperbárica de acrílico para animais de pequeno porte. Acta Cir Bras. 2001; 16:26770.

19. Pereira MLL. Câmara hiperbárica de acrílico para animais de pequeno porte [Tese - Mestrado]. Universidade Federal de São Paulo - Escola Paulista de Medicina; 1996.

20. Guimarães FAG, Taha MO, Simões MJ, Fagundes DJ. Ischemia reperfusion of the small intestine and hyperbaric oxygen treatment: a morphologic study in rats. Transplant Proc. 2002; 34:977-9.
Correspondência:

Maria Ligia Lira Pereira

Rua Dr. Batista Pereira, 265

11015-101 Santos - SP

ligia.lyra@uol.com.br
Conflito de interesse: nenhum

Fonte de financiamento: nenhuma

Recebimento: 14/08/2004

Revisão: 26/09/2004

Aprovação: 02/11/2004

\section{Como citar este artigo:}

Pereira MLL, Scheidt TC, Simões MJ, Mosquette R, Gomes PO. Oxigenoterapia hiperbárica em lesões actínicas de colo de ratas: aspectos morfológicos e morfométricos. Acta Cir Bras. [serial online] 2004 Nov-Dez;19(6). Disponível em URL: http:// www.scielo.br/acb [também em CD-ROM].

*Figuras coloridas disponíveis em www.scielo.br/acb 